\title{
Design and characterization of SiON integrated optics components for optical coherence tomography
}

\author{
V. Duc Nguyen ${ }^{1}$, J. Kalkman¹, N. Ismail², F. Sun ${ }^{2}$, K. Wörhoff ${ }^{2}$, A. Driessen², \\ M. Pollnau ${ }^{2}$, and T.G. van Leeuwen ${ }^{1,3}$ \\ ${ }^{1}$ University of Amsterdam, Academic Medical Center, Biomedical Engineering \& Physics, Amsterdam, 1100 DE, The Netherlands \\ ${ }^{2}$ University Twente, Integrated Optical MicroSystems, Enschede, 7500 AE, The Netherlands \\ ${ }^{3}$ University of Twente, Biomedical Technology Institute, Biophysical Engineering, Enschede, 7500 AE, The Netherlands
}

Optical coherence tomography (OCT) is a technique for high resolution imaging of biological tissues with a depth range of a few millimeters [1]. OCT is based on interferometry to enable depth ranging. Currently, optical components for OCT are rather bulky and expensive; the use of integrated optical circuits presents a great opportunity to reduce costs and enhance system functionality and performance. We present the design and characterization of SiON-based [2] integrated optics waveguides, splitters, couplers and interferometers for OCT operating at a wavelength of $1.3 \mu \mathrm{m}$.

Optical field calculations (FieldDesigner, Phoenix Corp.) are performed to determine the range of channel widths required for single mode operation of SiON waveguides. For these single-mode waveguides, beam propagation method simulations (OptoDesigner, Phoenix Corp.) are performed on various optical structures. For OCT the power splitting ratio of the sample and reference arm is an important parameter to enhance the signal to noise ratio. We calculate splitting ratios for symmetric and asymmetric waveguide $\mathrm{Y}$ splitters. The focusing or collimation of the beam that exits the sample arm waveguide can be used to increase the amount of light present in the OCT measurement depth range and to increase the lateral imaging resolution. We calculate the focusing properties of elliptic waveguide couplers (see Fig.1).

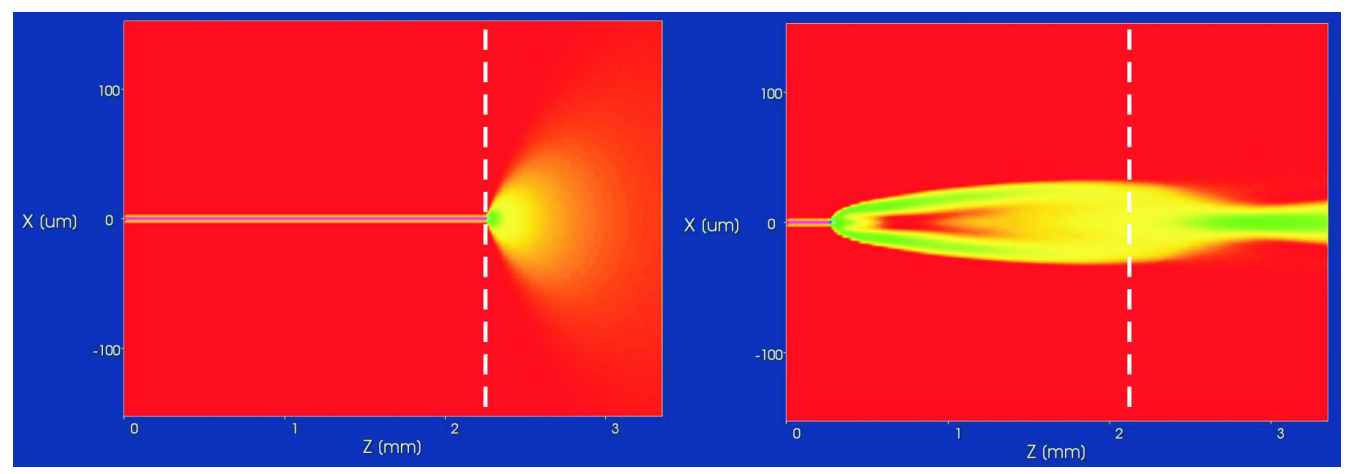

Fig. 1. Field profile for a straight waveguide (left) and for a waveguide with an elliptic output coupler (right).

The edge of the sample is indicated by a dashed white line. The diverging field distribution is transformed into a focused field distribution.

Interferometric measurements are performed on single straight waveguides in combination with a circulator (Fizeau/common-path geometry). The interferometric measurements are performed in the spectraldomain using either a super luminescent diode in combination with a spectrometer, or using a high speed scanning light source (HSL-2000, Santec Corp.) in combination with a point detector. In a similar way, measurements in the Michelson geometry are performed. The integrated optics OCT system performance are characterized in terms of signal drop off in depth, spatial resolution and signal to noise ratio.

\footnotetext{
${ }^{1}$ D. Huang, E.A. Swanson, C.P. Lin, J.S. Schuman, W.S. Stinson, W. Chang, M.R. Hee, T. Flotte, K. Gregory, C.A. Pullafito,and J. G. Fujimoto,"Optical Coherence Tomography”, Science. 254, 1178 (1991)

${ }^{2}$ Kerstin Wörhoff, Chris G. H. Roeloffzen, René M. de Ridder, Alfred Driessen, and Paul V. Lambeck, "Design and Application of Compact and Highly Tolerant Polarization-Independent Waveguides”, IEEE J. Lightw. Technol. 25, 1276 (2007)
} 